\title{
ASOCIACIÓN ENTRE CRONOTIPO Y LA INGESTA NOCTURNA DE ALIMENTOS CON EL ÍNDICE DE MASA CORPORAL.
}

\author{
ASSOCIATION BETWEEN CHRONOTYPE AND NOCTURNAL FOOD INTAKE WITH BODY MASS INDEX.
}

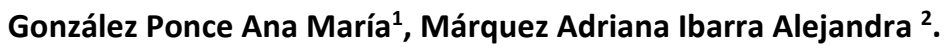

1 Universidad del Papaloapan campus Tuxtepec. 2 Universidad de Sonora campus Cajeme. México.

\begin{abstract}
RESUMEN
Introducción: El cronotipo, es un patrón regular de oscilación en procesos fisiológicos en relación con el ciclo de sueño-vigilia. La evidencia muestra que el cronotipo vespertino aunado a la ingestión de cena, tiene mayor riesgo de desarrollar obesidad. Objetivo: Determinar la asociación entre el cronotipo y la ingesta nocturna de alimentos, con el índice de masa corporal (IMC) y circunferencia de cintura. Material y Método: Estudio analítico transversal en universitarios $(n=264)$, las medidas antropométricas se obtuvieron por personal estandarizado, el cronotipo se obtuvo mediante el cuestionario MEQ-SA validado y adaptado al español, de Horne- Östberg y para la ingesta nocturna de alimentos, el cuestionario validado de MárquezSalazar. El análisis de datos se realizó con el paquete Stata versión 14, para todas las pruebas se consideró un valor $p<0.05$ como significancia estadística. Resultados: No se observó relación entre el IMC y los cronotipos ( $p=0.1136)$, tampoco se encontró asociación entre la circunferencia de cintura y el cronotipo $(p=0.1325)$, además no existió asociación $(p=0.709)$ entre las categorías de cronotipo y el hábito de alimentación nocturna. Conclusiones: No se encontró asociación entre las variables analizadas.
\end{abstract}

Palabras Clave: Ingesta de alimentos, Índice de Masa Corporal, Cronotipo.

\section{ABSTRACT}

Introduction: The chronotype is a regular pattern of oscillation in physiological processes in relation to the sleep-wake cycle. The evidence shows that the chronotype in the evening, together with the ingestion of dinner, has a higher risk of developing obesity. Objective: To determine of association between the chronotype and the nightly intake of food, with the body mass index (BMI) and waist circumference. Material and method: Transversal analytical study in university students ( $n=264)$, anthropometric measurements were obtained by standardized personnel, the chronotype was obtained by means of the MEQ- SA questionnaire validated and adapted to Spanish, by Horne- Östberg and for nighttime food intake, the validated questionnaire by Márquez-Salazar. The data analysis was performed with the Stata package version 14, for all tests a value $p<$ 0.05 was considered as statistical significance. Results: No relationship was observed between BMI and chronotypes ( $p=$ 0.1136), nor was there any association between waist circumference and chronotype $(p=0.1325)$, and there was no association $(p=0.709$ ) between chronotype categories and nighttime eating habits. Conclusions: No association was found between the variables analyzed.

Key words: Food intake, body mass index, Chronotype.

Correspondencia: Ana María González Ponce anamariagp25@hotmail.com

Recibido: 10 de mayo 2021, aceptado: 06 de octubre 2021

(C) Autor2022

(c) (i)

DOI: https://doi.org/10.29105/respyn21.1-2

Citation: González Ponce A.M., Márquez A.I. (2022) Asociación entre cronotipo y la ingesta nocturna de alimentos con el índice de masa corporal. Revista Salud Pública y Nutrición, 21 (1), 10-18. 


\section{Artículo Original}

\section{Introducción}

La obesidad es uno de los problemas de salud pública más importantes de México, reconocida como factor de riesgo en el desarrollo de las dos principales causas de mortalidad en adultos mexicanos. De acuerdo con la Encuesta Nacional de Nutrición y Salud (ENSANUT 2018), la prevalencia de obesidad en adultos fue de $36.1 \%$ (Barquera et al., 2020).

Aunque se considera una enfermedad de origen multifactorial, entre sus principales causas se encuentran el consumo de alimentos ricos en hidratos de carbono y/o grasas, el sedentarismo, la herencia y otros factores relacionados con el estilo de vida, como escasas horas de sueño (Guo X et al., 2013), y la ingesta nocturna de alimentos se han ligado con diversos indicadores de adiposidad (Bandín, 2014; Garaulet, 2014; Garaulet, 2013; Jakubowicz, 2013). Otro elemento que se ha asociado con la obesidad es el cronotipo o preferencia circadiana, que refleja el momento del día predilecto por un individuo para un ciclo de actividad/descanso. Cada individuo tiene características propias respecto a los ritmos circadianos, aunados a la hora de dormir, hora de levantarse y actividades diarias, lo que genera un rasgo estable en el tiempo (Lucassen et al., 2013) el cual depende de factores genéticos, edad, género y ambiente (Roenneberg et al., 2007; Miller et al., 2015). De esta manera se pueden distinguir individuos que se acuestan y levantan temprano (diurnos) en contraste a los que lo hacen más tarde (vespertinos/ nocturnos) (Roenneberg et al., 2007). Existe un tercer cronotipo (intermedio o neutro) al cual pertenece el $60 \%$ de la población adulta, en este caso los individuos se adaptan sin dificultad a cualquier horario (Kivelä L et al., 2018).

El cronotipo se ha relacionado con la ingesta de alimentos, particularmente en aquellos sujetos de tipo vespertino/nocturno, quienes mantienen una mayor ingesta de calorías durante la cena y por lo tanto un mayor riesgo de desarrollar alteraciones metabólicas como la obesidad (Miller et al., 2015; Escobar et al., 2016).

Así mismo se ha observado que en los estudiantes universitarios las preferencias vespertinas en conjunto con una restricción de sueño han sido asociadas a una dieta no saludable y ganancia de peso (Miller et al., 2015). Por lo anterior, el objetivo de este estudio fue determinar la asociación entre el cronotipo y la ingesta nocturna de alimentos con el índice de masa corporal (IMC) y circunferencia de cintura en universitarios de ambos sexos.

\section{Material y Método}

Diseño del estudio: Estudio analítico transversal. Población de estudio: estudiantes universitarios de una institución pública de Oaxaca, México en marzo $2018(n=264)$ con una mediana de edad de 21 años, $76.05 \%$ mujeres y $23.95 \%$ hombres, elegidos al azar que aceptaron participar en el estudio, mediante previa firma de consentimiento informado, aprobado por un comité de ética.

Criterios de inclusión: Estudiantes universitarios en activo durante el ciclo escolar 2018-2019- A de la Universidad del Papaloapan campus Tuxtepec, Oaxaca y que al momento de la medición de las variables a estudiar no consumieran fármacos para dormir y/o en tratamiento para la obesidad.

Criterios de eliminación: Contar con datos insuficientes de las variables de interés derivados a no querer responder los cuestionamientos o errores de captura.

Que al momento de la medición de las variables incumpliera con alguno de los criterios de inclusión. Selección de la muestra: Para la determinación del tamaño de la muestra se usó la fórmula para muestra de fracción grande con un nivel de confianza del 95 $\%$.

$$
n=\frac{N^{*} Z_{u}^{2} p^{*} q}{d^{2} *(N-1)+Z_{a}^{2} p^{*} q}
$$

Donde:

- $\mathrm{N}=$ Total de la población (840 estudiantes)

- $Z \alpha=1.96$ al cuadrado

- $\mathrm{p}=$ proporción esperada (en este caso $5 \%=0.05$ )

- $\mathrm{q}=1-\mathrm{p}$ (en este caso 1-0.05 = 0.95)

- $\mathrm{d}=$ precisión $(5 \%)$

La muestra resultante fue $n=264$

$\underline{\text { Recolección de los datos: }}$

Se realizó la recolección de los datos de interés en marzo de 2018 en el hospital robotizado de la Universidad del Papaloapan campus Tuxtepec, 


\section{Artículo Original}

Oaxaca, como parte de la recopilación de datos sobre salud en la población estudiantil vigente (840 alumnos). De ahí se seleccionó una muestra al azar de 264 estudiantes que cumplieran con los criterios de inclusión antes mencionados.

\section{Antropometría:}

Las medidas antropométricas se obtuvieron por personal entrenado y estandarizado a través de protocolos convencionales, usando técnicas y procedimientos internacionalmente aceptados (Shamah et al., 2006).

La circunferencia de cintura se midió con una cinta antropométrica marca SECA $®$ con capacidad de 150 $\mathrm{cm}$ para clasificar a los sujetos, utilizando los puntos de corte propuestos por la OMS (Organización Mundial de la Salud) de $>88 \mathrm{~cm}$ en mujeres y $>102$ $\mathrm{cm}$ en hombres, con alto riesgo cardiovascular (NOM-174-SSA1-1998).

La talla se midió con el uso de un estadímetro marca Seca ${ }^{\circledR}$ con capacidad de $220 \mathrm{~cm}$ y con una precisión de $1 \mathrm{~mm}$, y el peso corporal se obtuvo utilizando una balanza electrónica marca Tanita ${ }^{\circledR}$ (Modelo 1583, Tokio, Japón), con una precisión de 100 g. El IMC (kg/m2) se calculó para los sujetos con datos completos de talla y peso y se usaron como puntos de corte los propuestos por la OMS y señalados en la NOM-174-SSA1-1998.

\section{Cronotipo:}

El cronotipo se midió a través del cuestionario auto aplicado MEQ- SA, previamente validado y adaptado al español, el cual permite establecer el tiempo de sueño preferido del individuo clasificándolo como diurno, intermedio o vespertino/nocturno (Horne y Östberg, 1976). El cuestionario consta de 19 preguntas en las que se debe elegir una sola opción. Posteriormente se sumaron todos los puntos para determinar el cronotipo de acuerdo con los siguientes rangos: 5986 puntos diurno, $42-58$ puntos intermedio y 16-41 puntos vespertino o nocturno.

Se han diseñado varios cuestionarios para medir el cronotipo, sin embargo, el cuestionario MEQ es uno de los instrumentos más utilizados y aceptados para medir el cronotipo avalado por la Academia Americana de Medicina del Sueño. El MEQ está considerado como el estándar de oro para medir el cronotipo y se ha traducido a varios idiomas.

En estudios realizados en sujetos sanos han informado que el MEQ tiene una adecuada fiabilidad y validez (Di Milia et al., 2013; Lee et al., 2014; Roveda et al., 2017). El MEQ contiene 19 ítems relativos a los tiempos de sueño-vigilia y los tiempos preferidos para las actividades físicas y mentales, así como el estado de alerta subjetivo, mismos que se puntúan utilizando un formato de respuesta tipo Likert. Se ha sugerido que los puntos de corte consideren la edad, el sexo, las variaciones culturales, la estación de nacimiento y la pubertad (Adan y Natale, 2002; Caci et al, 2009; Kim et al, 2002). Una traducción al sueco del MEQ realizada por Ståleby y colaboradores informaron de una alta fiabilidad de la escala en una población adulta sana (Ståleby et al, 2016).

\section{Historia dietética:}

Para evaluar el comportamiento alimentario, solo en cuanto a la ingesta nocturna de alimentos, se utilizó el ítem correspondiente a dicho rubro, del cuestionario validado de Márquez- Salazar y coautores (Márquez et al., 2014). Dicho cuestionario ha sido validado en población universitaria mexicana con índices de reproducibilidad (ICC 0.76) y validez (alfa de Cronbach 0.98) satisfactorios de acuerdo a la Teoría de Respuesta al Ítem (TRI) para medir el comportamiento alimentario en estudiantes universitarios.

\section{Análisis estadístico:}

El análisis de los datos se realizó con el paquete estadístico Stata versión 14. Las variables continuas se muestran en medianas y percentiles 25 y 75 , derivado a su distribución no paramétrica. Las variables categóricas se muestran en frecuencias.

Para todas las pruebas se consideró un valor $\mathrm{p}<0.05$ como significancia estadística. Se realizaron pruebas X2 para las variables categóricas de interés y pruebas de correlación de Spearman para las variables continuas.

\section{Resultados}

Las características generales de los 263 alumnos objeto de estudio (se eliminó un dato por no contar con mediciones completas) se muestran en la tabla 1 y 2. De las variables de interés se encontró una mayor 
proporción de mujeres en comparación a los hombres (76.05\% vs $23.95 \%$ ), la mediana de edad fue de 21 años, de IMC 24.6 y el $49.81 \%$ de la muestra se encontraron en la categoría de normalidad para esta variable, ver tablas 1 y 2 .

Tabla 1. Características generales de la población*

\begin{tabular}{lrrr}
\hline \multicolumn{1}{c}{ Variable } & P25 & Mediana & P75 \\
\hline Edad (años) & 20 & 21 & 22 \\
Cintura $(\mathrm{cm})$ & 75.0 & 80.0 & 87.0 \\
Estatura $(\mathrm{m})$ & 1.5 & 1.6 & 1.63 \\
$\mathrm{IMC}(\mathrm{kg} / \mathrm{m} 2)$ & 22.4 & 24.6 & 27.8 \\
Peso $(\mathrm{kg})$ & 54.0 & 61.4 & 69.0 \\
\hline
\end{tabular}

Fuente:Encuesta

$* \mathrm{n}=263$

IMC= Índice de masa corporal, $\mathrm{Kg}=$ kilogramos, $\mathrm{cm}=$ centímetros,, $\mathrm{m}=$ metro.

P25 = percentil $25, \mathrm{P} 75=$ percentil 75

Tabla 2. Distribución de IMC y

\begin{tabular}{lrr}
\multicolumn{2}{c}{ Sexo } \\
\hline \multicolumn{1}{c}{ Variable } & $\mathrm{N}$ & $\%$ \\
\hline Sexo & & \\
Mujer & 200 & 76.1 \\
Hombre & 63 & 24.0 \\
Categoría de IMC & & \\
Bajo peso & 7 & 2.7 \\
Normal & 131 & 49.8 \\
Sobrepeso & 88 & 33.5 \\
Obesidad I & 30 & 11.4 \\
Obesidad II & 6 & 2.3 \\
Obesidad III & 1 & 0.4 \\
\hline Fuente: Encuesta
\end{tabular}

Fuente: Encuesta

$\mathrm{n}=263$

IMC= Índice de masa corporal.

Derivado de la encuesta para determinar el cronotipo, se observó que el $66.54 \%$ se encontraba en la categoría intermedia; respecto a las personas que respondieron tener el hábito de cenar, se determinó que el $76.43 \%$ de la población estudiada, respondió de manera afirmativa, ver tabla 3 .
Tabla 3. Cronotipo y hábitos de alimentación nocturna

\begin{tabular}{lrr}
\hline Variable & $\mathrm{N}$ & $\%$ \\
\hline Tipo de Cronotipo & 74 & 28.1 \\
Diurno & 175 & 66.5 \\
Intermedio & 14 & 5.3 \\
Nocturno & \\
Hábitos de alimentación nocturna & \\
Cena & & \\
Sí (diario) & 201 & 76.4 \\
No (diario) & 24 & 9.1 \\
Entre semana & 20 & 7.0 \\
Fin de semana & 18 & 6.8 \\
\hline
\end{tabular}

Fuente: Encuesta

$n=263$

Respecto a la relación entre el IMC como variable categórica y los diferentes tipos de cronotipo, no se observó una tendencia o relación entre estas dos variables, ver tabla 4; además al realizar la correlación de Spearman con las variables de forma continua, tampoco se encontró relación alguna, con un valor $\mathrm{p}=0.1136$; en este mismo sentido, al realizar la correlación de Spearman entre la circunferencia de cintura y el cronotipo, no se encontró una asociación con un valor $\mathrm{p}=0.1325$.

Tabla 4. Relación entre IMC y cronotipo

\begin{tabular}{lrrrrrr}
\hline & \multicolumn{7}{c}{ Tipo de cronotipo } \\
\cline { 2 - 7 } Categorías IMC & Diurno & \multicolumn{3}{c}{ Intermedio } & \multicolumn{3}{c}{ Nocturno } \\
& $\mathrm{n}$ & $\%$ & $\mathrm{n}$ & $\%$ & $\mathrm{n}$ & $\%$ \\
\hline Bajo peso & 0.0 & 0.0 & 7 & 2.7 & 0 & 0.0 \\
Normal & 34 & 12.9 & 88 & 33.5 & 9 & 3.4 \\
Sobrepeso & 24 & 9.1 & 60 & 22.8 & 4 & 1.5 \\
Obesidad I & 12 & 4.6 & 17 & 6.5 & 1 & 0.4 \\
Obesidad II & 4 & 1.5 & 2 & 0.8 & 0 & 0.0 \\
Obesidad III & 0.0 & 0.0 & 1 & 0.4 & 0 & 0.0 \\
\hline Fuente:Encuesta & & & & & & \\
n=263 & & & & & & \\
Chi ${ }^{2}$ p>.05 & & & & & & \\
IMC $=$ Índice de masa & & & & & &
\end{tabular}


La relación entre las categorías de cronotipo y los hábitos de alimentación nocturna, tampoco mostró una asociación entre las mismas, con un valor $\mathrm{p}=0.709$, ver tabla 5 .

Tabla 5. Relación entre IMC y hábitos de alimentación nocturna.

\begin{tabular}{|c|c|c|c|c|c|c|c|c|}
\hline \multirow{3}{*}{$\begin{array}{l}\text { Categoría } \\
\text { IMC }\end{array}$} & \multicolumn{8}{|c|}{ Hábitos de alimentación nocturna (cena) } \\
\hline & \multicolumn{2}{|c|}{$\begin{array}{c}\text { Entre } \\
\text { semana }\end{array}$} & \multicolumn{2}{|c|}{$\begin{array}{l}\text { Solo fines de } \\
\text { semana }\end{array}$} & \multicolumn{2}{|c|}{ No cenan } & \multicolumn{2}{|c|}{$\begin{array}{l}\text { Si, todos } \\
\text { los días }\end{array}$} \\
\hline & $\mathrm{n}$ & $\%$ & $\mathrm{n}$ & $\%$ & $\mathrm{n}$ & $\%$ & $\mathrm{n}$ & $\%$ \\
\hline Bajo peso & 0 & 0.0 & 1 & 0.4 & 1 & 0.4 & 5 & 1.9 \\
\hline Normal & 10 & 3.8 & 5 & 1.9 & 14 & 5.3 & 102 & 38.8 \\
\hline Sobrepeso & 9 & 3.4 & 10 & 3.8 & 6 & 2.3 & 63 & 24.0 \\
\hline Obesidad I & 1 & 0.4 & 1 & 0.4 & 2 & 0.8 & 26 & 9.9 \\
\hline Obesidad II & 0 & 0.0 & 1 & 0.4 & 1 & 0.4 & 4 & 1.5 \\
\hline Obesidad III & 0 & 0.0 & 0 & 0.0 & 0 & 0.0 & 1 & 0.4 \\
\hline \multicolumn{9}{|c|}{$\begin{array}{l}\text { Fuente: Encuesta } \\
n=263\end{array}$} \\
\hline $\begin{array}{l}\mathrm{Chi}^{2} \quad \mathrm{P}>.05 \\
\mathrm{IMC}=\text { Índice } \mathrm{c}\end{array}$ & s & & & & & & & \\
\hline
\end{tabular}

\section{Discusión}

A pesar de que una dieta poco saludable se ha asociado con la obesidad y que los sujetos con un cronotipo vespertino/nocturno se han relacionado con una ingesta nocturna elevada de alimentos, misma que podría predecir un mayor riesgo de obesidad entre dichos individuos en comparación con los del cronotipo matutino. En el presente estudio no se pudo demostrar la asociación entre el cronotipo, la ingesta nocturna de alimentos, con el IMC y la circunferencia de cintura, estos resultados comparten evidencia según lo encontrado por Hernández (Hernández et al., 2020), donde no se encontró asociación entre la calidad del sueño e indicadores antropométricos y de consumo alimentario del personal de la salud de la muestra estudiada.

En 2019, González y colaboradores en una muestra conformada por 50 enfermeras, revelaron que los valores de IMC no mostraron diferencias significativas $(\mathrm{p}<0.5)$ según el cronotipo de la población estudiada (González et al., 2019). En concordancia con lo anterior, De Amicis y colaboradores no encontraron asociación entre el cronotipo y la obesidad abdominal y la grasa visceral; pero no se investigó la interacción de la dieta en dicha asociación, lo mismo que ocurrió con la presente investigación (De Amicis et al., 2020). Este último aspecto fue examinado por investigadores finlandeses, quienes evidenciaron que el cronotipo no cambiaba la asociación entre una dieta equilibrada y la obesidad, concluyendo que la ingesta energética nocturna puede desempeñar un papel en la obesidad independientemente del cronotipo. (Maukonen et al., 2019).

En el presente estudio, no se evaluó la calidad de la dieta ni el aporte energético de la ingesta nocturna, lo cual explicaría la no asociación entre las variables evaluadas.

Zerón-Rugerio y colaboradores examinaron la asociación del cronotipo con una dieta saludable y la obesidad en adultos jóvenes entre 18-25 años, mostrando que los sujetos con cronotipo vespertino/nocturno, tienen menor adherencia a una dieta saludable, así como un mayor IMC, aunque no investigaron la obesidad abdominal a través de la medición de la circunferencia de cintura, como se realizó en la presente investigación (Zerón et al., 2019).

Por otra parte, es importante mencionar que respecto al cronotipo intermedio, Machado-Rojas y coautores concluyen que existe un claro predominio de este cronotipo, seguido en frecuencia por el matutino y el vespertino/nocturno. Lo que concuerda con los resultados obtenidos en esta investigación (Machado et al., 2019).

Dentro de las implicaciones de los hallazgos encontrados en este estudio cabe señalar que el porcentaje del cronotipo diurno fue mayor en comparación al nocturno, aunque la mediana de edad fue de 21 años; lo que indica que su cronotipo ya ha cambiado gradualmente o está en esa transición, del nocturno al intermedio para instalarse al final en el cronotipo diurno (Paine et al., 2006; Yu et al., 2015), esto probablemente también se deba a que al ser estudiantes de la salud, su cronotipo se ve afectado por las actividades que diariamente se llevan a cabo dentro de su programa académico. Además, se ha identificado la transición entre la adolescencia y la edad adulta como un período de mayor riesgo de aumento de peso excesivo, disminución de la calidad de la dieta y de la actividad física, independientemente del cronotipo (Nelson, 2018). 


\section{Artículo Original}

En relación a la ingesta nocturna de alimentos, los datos hallados en el presente estudio muestran que no hay diferencias entre las categorías de cronotipo, contrario a lo reportado por Roßbach y colaboradores en el 2018, al señalar que el cronotipo vespertino entre los adolescentes se asocia con un cambio en el consumo de alimentos hacia la cena y que por lo tanto, los patrones de alimentación de los adolescentes parecen seguir su reloj interno en lugar de horarios socialmente determinados (Roßbach et al., 2018). Sin embargo, cabe destacar que se trata de etapas de la vida en las que la preferencia diurna se retrasa, alcanzando su máximo alrededor de los 20 años (Roenneberg et al., 2007) y que los cambios en los patrones de consumo de alimentos, la exposición a la luz artificial durante la noche y el uso de dispositivos electrónicos las 24 horas del día, los 7 días de la semana, puede retrasar el inicio del sueño $\mathrm{y}$, por tanto, alterar los ritmos circadianos, emergiendo como un factor de riesgo potencial para la obesidad en este grupo de edad (Potter et al., 2016).

Por otra parte, en 2019, McMahon y colaboradores encontraron que los sujetos con cronotipo matutino, cuando se consideraba el número de horas de sueño ( $<6$ horas), eran más propensos a tener un mayor porcentaje de grasa corporal, relación cintura-cadera y relación cintura-altura en comparación con aquellos individuos con un cronotipo intermedio, además no se observaron relaciones entre la falta de sueño y la obesidad entre los sujetos con cronotipo vespertino/nocturno. En este estudio no se determinó el tiempo de sueño, variable que pudiera intervenir en la no asociación de las variables estudiadas, de ahí que las alteraciones metabólicas relacionadas con el sueño entre diferentes cronotipos merecen una mayor investigación (McMahon et al., 2019).

Xiao y colaboradores en el 2019, al investigar la relación entre el horario de comidas y el IMC dependiente del cronotipo, encontraron una asociación entre un mayor porcentaje de la ingesta diaria total de energía consumida durante la noche con mayor probabilidad de tener sobrepeso $\mathrm{u}$ obesidad, particularmente en personas con un cronotipo vespertino. Estas asociaciones fueron más fuertes para la ingesta de hidratos de carbono y proteínas que para la ingesta de grasas (Xiao et al., 2019).
En este estudio no se evaluó el contenido nutrimental y energético de la ingesta diaria y de la ingesta nocturna, sin embargo Lucassen y coautores, quienes sí evaluaron los contenidos nutrimentales y calóricos de la dieta, tampoco encontraron diferencias significativas en el número total de comidas diarias y en el tamaño de las porciones entre los cronotipos matutinos y vespertino/nocturno, además, el patrón vespertino se asoció con una mayor pérdida de grasa en comparación con la masa libre de grasa (Lucassen et al., 2013). Aunado a lo anterior, un estudio de ingesta dietética informó que la ingesta de alimentos a altas horas de la noche era menos saciante y provocaba una mayor ingesta total de energía (De Castro, 2004). En este sentido, los resultados de la evaluación de la sensación de hambre entre dos tipos de cronotipo, no pudieron identificar ninguna diferencia significativa entre los tipos matutino y vespertino/nocturno (Meule et al., 2012). No obstante, Muñoz y colaboradores revelaron una interacción significativa entre el cronotipo y el índice de masa corporal con respecto a la energía y los nutrimentos consumidos en la cena (Muñoz et al, 2017).

Este estudio presenta ciertas limitaciones ya que contempló el análisis de una muestra de universitarios, a los cuales no se les hicieron determinaciones de conducta alimentaria, de ingesta de nutrimentos, ni composición corporal completa, debido a que la recolección original de los datos implicaba aspectos y variables generales sobre la salud de los estudiantes.

Además, no se evaluó el contenido nutrimental y energético en específico de la ingesta nocturna de alimentos, tampoco se determinó el número de horas dedicadas al sueño, variables que pudieron influir en los resultados encontrados.

\section{Conclusiones}

Dadas las características de la población estudiada, existen más allá del cronotipo, otros aspectos como la alteración del sistema circadiano que repercuten en el sueño, la ingestión de alimentos y el gasto energético en dicha población. La ingestión de alimentos durante la noche y el recorte de las horas de sueño se han señalado como principales cronodisruptores, componentes que requieren de mayor investigación, además de la determinación del cronotipo como un criterio para la prevención y 
modificación de hábitos inadecuados que pueden incrementar el riesgo a desarrollar enfermedades metabólicas.

\section{Bibliografía}

Adan A, Natale V. (2002). Gender differences in morningness-eveningness preference. Chronobiol Int, 19 (4),709-20. https://doi:10.1081/CBI-120005390.

Bandín, C., Martinez-Nicolas, A., Ordovás, J. M., Madrid, J. A., \& Garaulet, M. (2014). Circadian rhythmicity as a predictor of weight-loss effectiveness. International journal of obesity (2005), 38(8), 1083-1088. https://doi.org/10.1038/ijo.2013.211

Barquera, S., Hernández-Barrera, L., Trejo-Valdivia, B., Shamah, T., Campos-Nonato, I., \& RiveraDommarco, J. (2020). Obesidad en México, prevalencia y tendencias en adultos. Ensanut 2018-19. Salud Pública de México, 62(6).

Caci H, Deschaux O, Adan A, Natale V. (2009). Comparing three morningness scales: age and gender effects, structure and cut-off criteria. Sleep Med. 10(2),240-45.

doi: 10.1016/j.sleep.2008.01.007.

De Amicis, R., Galasso, L., Leone, A., Vignati, L., De Carlo, G., Foppiani, A., Montaruli, A., Roveda, E., Cè, E., Esposito, F, Vanzulli, A., Battezzati, A. y Bertoli, S. (2020). ¿La distribución de la grasa abdominal está asociada con el cronotipo en adultos independientemente de los factores del estilo de vida? Nutrients, 12 (3), 592 .

https://doi.org/10.3390/nu12030592.

De Castro, J. M. (2004). The time of day of food intake influences overall intake in humans. The Journal of nutrition, 134(1), 104-111. https://doi: 10.1093/jn/134.1.104.

De la Federación, D. O. (2000). NORMA Oficial Mexicana NOM-174-SSA1-1998, Para el manejo integral de la obesidad. Rev méd IMSS, 38(5), 397-403.
Di Milia L, Adan A, Natale V, Randler C. (2013). Reviewing the psychometric properties of contemporary circadian typology measures. Chronobiol Int. 30(10):1261-71. https://doi:10.3109/07420528.2013.817415.

Escobar, C., Ángeles-Castellanos, M., Bautista, E. N. E., \& Buijs, R. M. (2016). Food during the night is a factor leading to obesity. Revista Mexicana de Trastornos Alimentarios, 7(1), 78-83. https://doi.org/10.1016/j.rmta.2016.01.001

Garaulet, M., Smith, CE, Gomez - Abellán, P., Ordovás - Montañés, M., Lee, YC, Parnell, LD... y Ordovás, JM (2014). La variante del gen circadiano REV - ERB - ALPHA se asocia con la obesidad en dos poblaciones independientes: el Mediterráneo y el norte de América. Investigación de alimentos y nutrición molecular, 58 (4), 821-829.

https://doi.org/10.1002/mnfr.201300361

González, J. A. O., Pliego, M. V., Nájera, J. A., Espinosa, C. B., Ruíz, J. A. C., \& Reboredo, T. B. (2019). Influencia del cronotipo en la composición corporal y variables metabólicas de enfermeras. Nutrición clínica y dietética hospitalaria, 39(4), 77-85. http://dx.doi.org/10.12873/3943ortega

Guo, X., Zheng, L., Wang, J., Zhang, X., Zhang, X., Li, J., \& Sun, Y. (2013). Epidemiological evidence for the link between sleep duration and high blood pressure: a systematic review and meta-analysis. Sleep medicine, 14(4), 324-332. https://doi.org/10.1016/j.sleep.2012.12.001

Hernández AJ., Rivera LG., López MM. (2020). Indicadores antropométricos y consumo alimentario del personal de salud según su turno laboral, cronotipo y calidad del sueño. Rev Cienc Salud, 19(2), 1-16.

https://doi.org/10.12804/revistas.urosario.edu.co /revsalud/a.10293

Horne, J. A., \& Östberg, O. (1976). A selfassessment questionnaire to determine morningness-eveningness in human circadian rhythms. International journal of chronobiology, 4,97- 110. 


\section{Artículo Original}

Jakubowicz, D., Barnea, M., Wainstein, J., \& Froy, O. (2013). High caloric intake at breakfast vs. dinner differentially influences weight loss of overweight and obese women. Obesity, 21(12), 2504-2512.

https:// doi: 10.1002/oby.20460

Kivelä L, Papadopoulos MR, Antypa N. (2018). Chronotype and Psychiatric Disorders. Curr Sleep Medicine Rep, 1(4),94-103. https:// doi: 10.1007/s40675-018-0113-8.

Kim S, Dueker GL, Hasher L, Goldstein D. (2002). Children's time of day preference: age, gender and ethnic differences. Personal Individ Differ, 33(7),1083-90.

https:// DOI: 10.1016/S0191-8869(01)00214-8

Lee JH, Kim SJ, Lee SY, Jang KH, Kim IS, Duffy JF. (2014). Reliability and validity of the Korean version of morningness- eveningness questionnaire in adults aged 20-39 years. Chronobiol Int, 31(4),479-86. https://doi:10.3109/07420528. 2013.867864.

Lucassen, E. A., Zhao, X., Rother, K. I., Mattingly, M. S., Courville, A. B., De Jonge, L., ... \& Sleep Extension Study Group. (2013). Evening chronotype is associated with changes in eating behavior, more sleep apnea, and increased stress hormones in short sleeping obese individuals. PloS one, 8(3), e56519. https://doi.org/10.1371/journal.pone.0056519

Machado Rojas, Arlan, \& Broche Pérez, Yunier, \& Norman Montenegro, Osvaldo, \& de la Torre Santos, María Elena, \& Tirado Saura, Daniel, \& Machado de la Torre, Carolina (2019). Caracterización de la tipología circadiana en estudiantes universitarios en relación con el tipo de carrera. MediSur, 17 (2), 284-289.

https://www.redalyc.org/articulo.oa?id=1800612 12018

Márquez-Sandoval, Y. F., Salazar-Ruiz, E. N., Macedo-Ojeda, G., Altamirano-Martínez, M. B., Bernal-Orozco, M. F., Salas-Salvadó, J., \& Vizmanos-Lamotte, B. (2014). Diseño y validación de un cuestionario para evaluar el comportamiento alimentario en estudiantes mexicanos del área de la salud. Nutrición hospitalaria, 30(1), 153-164.

https://dx.doi.org/10.3305/nh.2014.30.1.7451

Maukonen, M., Kanerva, N., Partonen, T., \& Männistö, S. (2019). Chronotype and energy intake timing in relation to changes in anthropometrics: a 7-year follow-up study in adults. Chronobiology international, 36(1), 2741.

https://doi.org/10.1080/07420528.2018.1515772

McMahon, D. M., Burch, J. B., Youngstedt, S. D., Wirth, M. D., Hardin, J. W., Hurley, T. G., Blair, S. N., Hand, G. A., Shook, R. P., Drenowatz, C., Burgess, S., \& Hebert, J. R. (2019). Relationships between chronotype, social jetlag, sleep, obesity and blood pressure in healthy young adults. Chronobiology international, 36(4), 493-509. https://doi.org/10.1080/07420528.2018.1563094

Meule, A., Roeser, K., Randler, C., \& Kübler, A. (2012). Skipping breakfast: morningnesseveningness preference is differentially related to state and trait food cravings. Eating and weight disorders: EWD, 17(4), e304-e308.

https://doi.org/10.3275/8723

Miller, A. L., Lumeng, J. C., \& LeBourgeois, M. K. (2015). Sleep patterns and obesity in childhood. Current opinion in endocrinology, diabetes, and obesity, 22(1), 41.

doi: 10.1097/MED.0000000000000125

Muñoz, J. S. G., Cañavate, R., Hernández, C. M., Cara-Salmerón, V., \& Morante, J. J. H. (2017). The association among chronotype, timing of food intake and food preferences depends on body mass status. European journal of clinical nutrition, 71(6), 736-742.

https:// doi: 10.1038/ejcn.2016.182.

Nelson, M. C., Story, M., Larson, N. I., NeumarkSztainer, D., \& Lytle, L. A. (2008). Emerging adulthood and college-aged youth: an overlooked age for weight-related behavior change. Obesity (Silver Spring, Md.), 16(10), 2205-2211. https://doi.org/10.1038/oby.2008.365

Paine, S. J., Gander, P. H., \& Travier, N. (2006). The epidemiology of morningness/eveningness: 
influence of age, gender, ethnicity, and socioeconomic factors in adults (30-49 years). Journal of biological rhythms, 21(1), 68-76. https:// doi: 10.1177/0748730405283154.

Potter, G. D., Skene, D. J., Arendt, J., Cade, J. E., Grant, P. J., \& Hardie, L. J. (2016). Circadian Rhythm and Sleep Disruption: Causes, Metabolic Consequences, and Countermeasures. Endocrine reviews, 37(6), 584-608.

https://doi.org/10.1210/er.2016-1083

Roenneberg, T., Kuehnle, T., Juda, M., Kantermann, T., Allebrandt, K., Gordijn, M., \& Merrow, M. (2007). Epidemiology of the human circadian clock. Sleep Medicine Reviews, 11(6), 429-438. https://doi.org/10.1016/j.smrv.2007.07.005

Roßbach, S., Diederichs, T., Nöthlings, U., Buyken, A. E., \& Alexy, U. (2018). Relevance of chronotype for eating patterns in adolescents. Chronobiology international, 35(3), 336-347. https:// doi: 10.1080/07420528.2017.1406493.

Roveda E, Vitale J, Montaruli A, Galasso L, Carandente F, Caumo A. (2017). Predicting the actigraphy-based acrophase using the Morningness-Eveningness Questionnaire (MEQ) in college students of North Italy. Chronobiol Int. 34 (5):551-62.

https://doi:10.1080/07420528.2016.1276928.

Sateia, M. J. (2014). International classification of sleep disorders. Chest, 146(5), 1387-1394.

Shamah- Levy T, Villalpando- Hernández S, RiveraDommarco J. (2006) Manual de Procedimientos para Proyectos de Nutrición. Cuernavaca, México. Instituto Nacional de Salud Pública.

Ståleby M, Steingrimsson S, Ioannou M. (2016). Swedish translation and reliability of the Morningness-Eveningness Questionnaire. Eur Psychiatry. 33(Supplement), S524.

https:// doi: 10.1016/j.eurpsy.2016.01.1530

Xiao, Q., Garaulet, M., \& Scheer, F. A. (2019). Meal timing and obesity: Interactions with macronutrient intake and chronotype. International Journal of Obesity, 43(9), 17011711. https:// doi: 10.1038/s41366-018-0284

Yu, J. H., Yun, C. H., Ahn, J. H., Suh, S., Cho, H. J., Lee, S. K... \& Baik, S. H. (2015). Evening chronotype is associated with metabolic disorders and body composition in middle-aged adults. The Journal of Clinical Endocrinology \& Metabolism, 100(4), 1494-1502. https://doi: 10.1210/jc.2014-3754

Zerón-Rugerio, M.F.; Cambras, T.; IzquierdoPulido, M. (2019). Social jet lag associates negatively with the adherence to the mediterranean diet and body mass index among young adults. Nutrients, $11,1756$. https://doi: 10.3390/nu11081756. 